\title{
Luftgetragene, organische Stoffe in Blattorganen
}

\author{
- Vorgang der ad-/absorptiven Anreicherung
}

\section{K. Figge}

NATEC Institut für Naturwissenschaftlich-Technische Dienste GmbH, Behringstr. 154, W-2000 Hamburg 50

Zusammenfassung. Ziel dieser Arbeit ist es, den die Schadwirkung einleitenden Schritt, d.h. die Akkumulation luftgetragener, organischer Stoffe durch Blattorgane (von Fichtennadeln) zu analysieren. Einbezogen werden dabei die in ihren physikalischchemischen Eigenschaften unterschiedlichen Stoffe Tetrachlorethen (TCE) und Furfural. Auch die Desorption beider Stoffe von vorher belasteten Fichtenzweigen wird berücksichtigt.

Die Ergebnisse aus den Versuchen mit TCE zeigen, daß zwischen der TCE-Konzentration in Fichtennadeln und der in der umgebenden Atmosphäre ein direkter, linearer Zusammenhang besteht, der mathematisch beschrieben werden kann. Der Vorgang der Desorption des TCE von Fichtenzweigen läßt sich in zwei verschieden schnelle Teilprozesse 1 . Ordnung zerlegen - in die Desorption von den Nadeln und die von den übrigen Zweigteilen - und ist ebenfalls mathematisch formulierbar.

Die TCE-Rückstandskonzentration in den Nadeln (Äquivalentkonzentration nach Desorption) hängt gleichfalls in linearer Weise von der TCE-Konzentration in der Atmosphäre ab und steigt mit fortschreitender Belastungszeit.

Im Gegensatz zum TCE tritt das Furfural vollständig - ohne daß sich ein meßbares Verteilungsgleichgewicht einstellt - aus der Atmosphäre auf die Fichtenzweige über, solange deren Aufnahmekapazität ausreicht. Es wird bevorzugt in den Nadeln festgelegt und ist danach nur noch zu einem geringen Anteil desorbierbar.

\section{Einleitung}

Der Übertritt von organischen Stoffen aus der Luft in Blattorgane $^{1}$ kann - ebenso wie der anorganischer Stoffe auf zwei Wegen erfolgen, d.h. durch geöffnete Stomata ${ }^{2}$ und/oder durch die Kutikula. Bei lipophilen Stoffen ist dem Transport durch die Kutikula besondere Aufmerksamkeit zu schenken.

Die Kutikula bildet die Grenzfläche zwischen Blattorgan und Atmosphäre. Sie ist als eine zusammenhängende, extrazelluläre Membran den äußeren Wänden der Epidermiszellen des Blattorgans aufgelagert und meist nur in bestimmten Bereichen des Blattorgans durch Stomata unterbrochen.
Aufgrund des lipophilen Charakters der Komponenten der Blattkutikula ist zu erwarten, daß diese eine besondere Affinität zu den lipophilen Stoffen in der Atmosphäre besitzt (RIEDERER, SCHÖNHERR 1984, 1985, 1986; KERLER, RIEDERER, SCHÖNHERR, 1984; SHAFER, SCHÖNHERR 1985; KERLER 1986; HARNISCH et al., 1983; Miller et al., 1985). Demnach stellt die pflanzliche Kutikula ein bedeutendes Akkumulationskompartiment für lipophile Organika dar.

Zur näheren Untersuchung des Vorganges der ad-/absorptiven Anreicherung von luftgetragenen Organika in Blattorganen wurde von uns beispielhaft ein Vertreter aus der Verbindungsklasse der aliphatischen Chlorkohlenwasserstoffe - das Tetrachlorethen (TCE) - und ein Mitglied aus der Verbindungsklasse der Aldehyde - das Furfural (eigene Abkürz.: F) - ausgewählt.

Aliphatische Chlorkoblenwasserstoffe sind bis auf wenige Ausnahmen anthropogenen Ursprungs und haben im Vergleich zu anderen organischen/anorganischen Stoffen relativ lange atmosphärische Halbwertzeiten, die zwischen wenigen Wochen (Trichlorethen) und mehreren Jahren (1,1,1-Trichlorethan) liegen (PEARSON 1982). Messungen aus den vergangenen 10 bis 15 Jahren (vgl. LIESENFELD, FIGGE, DOMMRÖSE 1990; DOMMRÖSE, FIGGE 1988) zeigen, daß z.B. die Konzentrationen an Tri- und Tetrachlorethen auch in der Luft der ruralen Gebiete von Industriestaaten stets zunahmen und regional Werte $>5 \mu \mathrm{g} / \mathrm{m}^{3}$ erreichten.

Aldebyde finden aufgrund ihrer chemischen Eigenschaften ebenfalls in der Technik vielfältige Einsatzmöglichkeiten. Sie gelangen in erheblicher Menge in die Umwelt und sind im Gegensatz zu den Vertretern der chlorierten Aliphaten wegen ihrer hohen Reaktivität in der Atmosphäre relativ kurzlebig.

Diese beiden Teststoffe wurden in ${ }^{14} \mathrm{C}$-markierter Form unter definierten Bedingungen dampfförmig mit vitalen Fichtenzweigen in Kontakt gebracht ( $\rightarrow$ Abschnitt 2).
1 Vgl. auch „Organische Luftschadstoffe und ihre Bedeutung für die terrestri-
sche Vegetation", UWSF 1 (2) $32-41(1990)$
2 Stomata: Verschließbare Spaltöffnungen in Blättern, die Transpiration und Gaswechsel der Pflanze regulieren 


\section{Experimentelle Angaben}

Zur Untersuchung des Ad-/Absorptions- und Desorptionsverhaltens der Teststoffe ${ }^{14} \mathrm{C}$-TCE und ${ }^{14} \mathrm{C}$-F an lebenden Fichtenzweigen wurden sechsjährige, eingetopfte Ramets eines definierten Fichtenklons eingesetzt.

Versuchsprinzip: Das Ende eines vitalen Fichtenzweiges ( 3 Nadeljahrgänge) wurde in die 25,6 I fassende Glasküvette der Begasungseinheit eingeschlossen, in deren Innenatmosphäre man danach durch einmalige oder aber wiederholte Stoffapplikation eine definierte Menge an gasförmigem ${ }^{14} \mathrm{C}$-TCE bzw. ${ }^{14} \mathrm{C}-\mathrm{F}$ verteilte und während der Begasungsdauer eine $\mathrm{CO}_{2}$-Konzentration von $0,03 \mathrm{Vol} . \%$ aufrecht hielt.

Während der 24- bis 72-stündigen Begasung des Zweiges in der Glasküvette, die ständig auf $22^{\circ} \mathrm{C} \pm 1{ }^{\circ} \mathrm{C}$ temperiert war, wurden in vorgegebenen Zeitintervallen über ein Septum jeweils 3 Luftproben zur radioanalytischen Bestimmung der Konzentration an ${ }^{14} \mathrm{C}-\mathrm{TCE}$ bzw. ${ }^{14} \mathrm{C}$-F entnommen. Nach 24, 48 bzw. $72 \mathrm{~h}$ wurde dann der Zweig aus der Begasungsküvette in den Glaszylinder der Desorptionseinheit überführt. Dabei wurden ihm Nadeln zur radioanalytischen Bestimmung der ad-/absorbierten Menge an Testsubstanz entnommen. Durch den Desorptionszylinder strömte ein konstanter Luftstrom $(90,5 \mathrm{ml} / \mathrm{min})$, durch den die von Nadeln und anderen Zweigteilen während der Begasungsphase aufgenommene Menge an Testsubstanz nach und nach wieder desorbiert wurde. Die Luft strömte nach dem Passieren des Desorptionszylinders durch mehrere hintereinander geschaltete Waschflaschen, die mit einer auf ca. $-10^{\circ} \mathrm{C}$ gekühlten Szintillatorflüssigkeit (L.S.C.-Cocktail „,Opti-Fluor“, Fa. Packard bzw. Tetralin-Szintillatorflüssigkeit, NATEC) und mit einer auf ca. $+1^{\circ} \mathrm{C}$ gekühlten, wäßrigen $2 \mathrm{n} \mathrm{KOH}$-Lösung gefüllt waren. In bestimmten Zeitabständen, die der Desorptionsgeschwindigkeit angepaßt waren, wurden den Waschflaschen jeweils 2 Flüssigkeitsproben entnommen und zur radioanalytischen Bestimmung der in ihnen angereicherten Mengen an Teststoff und/oder Metaboliten verwendet. Die Waschflaschen wurden sicherheitshalber nach jeweils $24 \mathrm{~h}$ durch neue mit frischen Absorberflüssigkeiten ersetzt.

Nach 99 h oder mehr wurde die Desorption beendet, der Zweig aus dem Desorptionszylinder gezogen und erneut Nadelproben zur radioanalytischen Bestimmung der Rückstandskonzentration an Teststoff genommen.

Die ganze Fichte wurde dann für weitere 28 bis 89 Tage an die freie Atmosphäre gebracht und danach wiederholt die Konzentration an Testsubstanz in den Nadeln, der Rinde ( + Bast) und dem Holz des belasteten $Z$ weiges radioanalytisch ermittelt.

Nach diesem Prinzip wurden mit $\left[1,2-{ }^{14} \mathrm{C}\right]$ Tetrachlorethen insgesamt 7 und mit [Carbonyl- ${ }^{14} \mathrm{C}$ ] Furfural insgesamt 6 verschiedene Begasungs- und Desorptionsversuche an Fichtenzweigen durchgeführt $(\rightarrow$ Tabellen 1, 2).

Tabelle 1: Begasung vitaler Fichtenzweige mit ${ }^{14} \mathrm{C}$-markiertem Tetrachlorethen $\left({ }^{14} \mathrm{C}\right.$-TCE) - Versuchsparameter

\begin{tabular}{|c|c|c|c|c|c|c|c|c|}
\hline \multirow{3}{*}{$\begin{array}{l}\text { Versuch } \\
\text { Nr. T- }\end{array}$} & \multicolumn{3}{|c|}{$\begin{array}{c}\text { Applikation an } \\
\left.{ }^{14} \mathrm{C} \text {-markiertem TCE }\right)\end{array}$} & \multicolumn{2}{|c|}{ Eingesetzter Zweig } & \multirow{3}{*}{$\begin{array}{l}\text { Begasungs- } \\
\text { dauer } \\
\text { [h] }\end{array}$} & \multicolumn{2}{|c|}{$\begin{array}{l}\text { TCE-Gleichgewichtskonzentration } \\
\text { in der Begasungsluft }\end{array}$} \\
\hline & & & & Gesamtgewicht & Nadelgewicht & & berechnet & gefunden \\
\hline & $\left.\{\mu \mid\}^{a}\right)$ & $\underline{=}$ & [mg] & [g] & [g] & & \multicolumn{2}{|c|}{$\left[\mu \mathrm{g} / \mathrm{dm}^{3}\right]$} \\
\hline 1 & 0,22 & & 0,357 & 28,1 & 15,5 & 24 & 13,98 & 10.13 \\
\hline II & 0,88 & & 1,429 & 23,2 & 14,2 & 24 & 55,93 & 39,29 \\
\hline III & 2,2 & & 3,57 & 22,9 & 12,7 & 24 & 139,8 & 100,4 \\
\hline IV & 4,4 & & 7,15 & 19,3 & 9,8 & 24 & 279,7 & 189,3 \\
\hline V & 4,4 & & 7,15 & 21,8 & 8,3 & 48 & 279,7 & 220,0 \\
\hline VI & $\left.13,2^{b}\right)$ & & 21,45 & 31,6 & 20,7 & 72 & $\left.839,1^{c}\right)$ & $\left.602,5^{c}\right)$ \\
\hline VII & $\left.13,2^{b}\right)$ & & 21,45 & 35,1 & 21,2 & 72 & $\left.839,1^{c}\right)$ & $\left.574,5^{c}\right)$ \\
\hline
\end{tabular}

a) Spez. Gewicht (Dichte): 1,624 ; spez. Radioaktivitäten der eingesetzten ${ }^{14} \mathrm{C}$-TCE-Chargen: Versuch $\mathrm{T}-\mathrm{I}$ bis $\mathrm{T}-\mathrm{IV} 13,03 \mu \mathrm{C} / \mathrm{mg} \hat{\mathrm{g}} 482,11 \mathrm{kBq} / \mathrm{mg}, \mathrm{Ver}-$ such $T-V$ bis $T-V I I 25,27 \mu \mathrm{Ci} / \mathrm{mg} \cong 934,99 \mathrm{kBq} / \mathrm{mg}$; die TCE-Mengen wurden verdampft und mit einem Luftstrom in das Begasungsgefäß transportiert b) Im Gegensatz zur Einfachapplikation in den Versuchen $T-I$ bis $T-V$ wurden diese ${ }^{14} \mathrm{C}$-TCE-Mengen in drei $4,4 \mu \mathrm{l}$-Portionen zu den Zeitpunkten 0,24 und $48 \mathrm{~h}$ appliziert

c) Gieichgewichtskonzentrationen jeweils nach der $3 .{ }^{14} \mathrm{C}$-TCE-Applikation, vgl. Fußnote b)

Tabelle 2: Begasung vitaler Fichtenzweige mit ${ }^{14} \mathrm{C}$-markierten Furfural $\left({ }^{14} \mathrm{C}-\mathrm{F}\right)$ - Versuchsparameter

\begin{tabular}{|c|c|c|c|c|c|c|c|c|}
\hline \multirow{3}{*}{$\begin{array}{l}\text { Versuch } \\
\text { Nr. F- }\end{array}$} & \multicolumn{3}{|c|}{$\begin{array}{c}\text { Applikation an } \\
{ }^{14} \mathrm{C} \text {-markiertem Furfurala) }\end{array}$} & \multicolumn{2}{|c|}{ Eingesetzter Zweig } & \multirow{3}{*}{$\begin{array}{l}\text { Begasungs- } \\
\text { dauer } \\
{[\mathrm{h}]}\end{array}$} & \multicolumn{2}{|c|}{$\begin{array}{l}\text { F-Gleichgewichtskonzentration } \\
\text { in der Begasungsluft }\end{array}$} \\
\hline & & & & \multirow{2}{*}{$\begin{array}{l}\text { Gesamtgewicht } \\
\text { [g] }\end{array}$} & \multirow{2}{*}{$\begin{array}{l}\text { Nadelgewicht } \\
\text { [g] }\end{array}$} & & berechnet & gefunden \\
\hline & {$[\mu l]^{\mathrm{a})}$} & $\Leftrightarrow$ & {$[\mathrm{mg}]$} & & & & \multicolumn{2}{|c|}{$\left[\mu \mathrm{g} / \mathrm{dm}^{3}\right]$} \\
\hline 1 & 6,2 & & 7,19 & 14,5 & 6,8 & 24 & 281,3 & 3,27 \\
\hline 11 & 6,2 & & 7,19 & 21,1 & 11,9 & 24 & 281,3 & 2,51 \\
\hline III & $\left.18,6^{b}\right)$ & & 21,57 & 29,5 & 19,6 & 72 & $\left.843,8^{c}\right)$ & $\left.33,3^{9}\right)$ \\
\hline IV & $\left.18,6^{b}\right)$ & & 21,57 & 19,7 & 10,2 & 72 & $\left.843,8^{c}\right)$ & $\left.14,1^{c}\right)$ \\
\hline v & 9,3 & & 10,78 & 21,2 & 11,6 & 24 & 421,9 & 10,6 \\
\hline VI & 9,3 & & 10,78 & 31,0 & 18,7 & 24 & 421,9 & 4,36 \\
\hline
\end{tabular}

a) Spez. Gewicht (Dichte): 1,159; spez. Radioaktivitäten der eingesetzten ${ }^{14} \mathrm{C}$-F-Chargen: Versuch $\mathrm{F}-1$ bis $\mathrm{F}-\mathrm{III} 24,36 \mu \mathrm{Ci} / \mathrm{mg} \cong 901,32 \mathrm{kBq} / \mathrm{mg}$, Versuch $\mathrm{F}-\mathrm{N}$ bis $\mathrm{F}-\mathrm{VI} 22,67 \mu \mathrm{Ci} / \mathrm{mg} \hat{\neq} 838,79 \mathrm{kBq} / \mathrm{mg}$; die Furfural-Mengen wurden verdampft und mit einem Luftstrom in das Begasungsgefäß transportiert

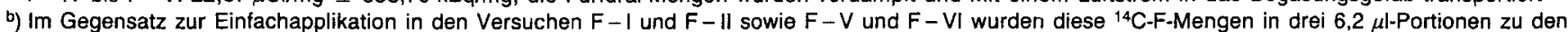
Zeitpunkten 0,24 und $48 \mathrm{~h}$ appliziert

c) Gleichgewichtskonzentrationen jeweils nach der 3. ${ }^{14} \mathrm{C}$-F-Applikation, vgl. FuBnote b) 


\section{Ergebnisse und Diskussion}

Wie FrANK et al. (1986) feststellten, haben aliphatische Chlorkohlenwasserstoffe große $\mathrm{Nadel} /$ Luft-Verteilungskoeffizienten. Selbst wenn diese auf die Frischmasse der Fichtennadeln bezogen werden, ist der Anreicherungseffekt für die Chlorkohlenwasserstoffe beträchtlich. FRANK et al. zeigten außerdem, daß die Geschwindigkeit der Gleichgewichtseinstellung für Chlorkohlenwasserstoff im Kontaktsystem , Nadel/Luft" besonders groß ist: unter vergleichbaren Bedingungen vom Zweig getrennte Fichtennadeln nahmen in der Anfangsphase ca. fünfmal schneller als Bienenwachs Chlorkohlenwasserstoffe aus der Luft auf.

Wir können diese Angaben aufgrund der Versuchsergebnisse ( $\rightarrow$ Abschnitt 2 ) bestätigen: unabhängig von der jeweils applizierten Menge hatte bereits eine Stunde nach Einlaß des ${ }^{14} \mathrm{C}$-TCE dessen Konzentration in der Gasphase einen konstant bleibenden Wert erreicht $\left(c_{g}\right)$; d.h., der Zweig nahm keine weitere durch diese indirekte Methode meßbare Menge an ${ }^{14} \mathrm{C}-\mathrm{TCE}$ mehr auf.

Die Tabellen $3+4$ zeigen, daß

1. mit zunehmender TCE-Gleichgewichtskonzentration in der Luft der Begasungsküvette auch die Konzentration an TCE in den Nadeln anstieg;
2. auch die Höhe der TCE-Rückstandskonzentration, die nach der Desoprtion in den Nadeln vorlag, in gleicher Weise von der anfangs applizierten TCE-Menge abhing.

Diese Abhängigkeit der TCE-Gleichgewichts- bzw. TCERückstandskonzentration in Fichtennadeln von der TCEGleichgewichtskonzentration im Luftraum (nach 24-stündiger Begasung) wird durch die Kurven in Abb. 1 veranschaulicht. Die obere Kurve gibt die Abhängigkeit der TCE-Konzentration $c_{n}$ in den begasten Nadeln von der TCE-Gleichgewichtskonzentration $c_{g}$ im Luftraum wieder. Es gilt hier mit sehr guter Korrelation

$$
c_{n}=0,103 \cdot c_{g}
$$

Gleichung (1): Zusammenhang zwischen der TCE-Konzentration in Fichtennadeln $\left(c_{n}\right)$ und der TCE-Gleichgewichtskonzentration in der Umgebungsluft $\left(c_{\mathrm{g}}\right)$

Hieraus muß geschlossen werden, daß sich im gesamten untersuchten Konzentrationsbereich - von $10 \mu \mathrm{g}$ TCE bis $189 \mu \mathrm{g}$ TCE pro 1 Luft - während der Belastung des Zweiges in der mit TCE beaufschlagten Atmosphäre schnell ein echtes Gleichgewicht eingestellt hat innerhalb einer Stunde.

Darüber hinaus zeigen die Befunde, daß man sich unter den gewählten Versuchsbedingungen noch in dem für kleine

Tabelle 3: TCE-Konzentrationen in den Nadeln der Fichtenzweige, die durch einmalige bzw. mehrfach wiederholte Eindosierung von gasförmiger Testsubstanz in die Versuchsvorrichtung begast und anschließend im definierten Luftstrom sowie an freier Luft desorbiert wurden (TCE-Äquivalentkonzentrationen)

\begin{tabular}{|c|c|c|c|c|c|}
\hline \multirow{2}{*}{$\begin{array}{c}\text { TCE-Begasungsversuch } \\
\text { (vgl. Tab. 1) } \\
\text { Nr. T- }\end{array}$} & \multirow{2}{*}{$\begin{array}{c}\text { zu Begasungsende } \\
\left.[\mu \mathrm{g} / \mathrm{g}]^{\mathrm{c}}\right)\end{array}$} & \multicolumn{4}{|c|}{$\begin{array}{c}\text { Konzentration an TCE in Fichtennadeln } \\
\left.\text { Iach 1. Desorption }{ }^{a}\right) \\
\text { nach 1. Desorption, Freilano } \\
\left.\text { und Nachdesorption }{ }^{b}\right)\end{array}$} \\
\hline & & $\left.[\mu \mathrm{g} / \mathrm{g}]^{C}\right)$ & $\left.[\%]^{d}\right)$ & $\left.[\mu \mathrm{g} / \mathrm{g}]^{c}\right)$ & $\left.[\%]^{d}\right)$ \\
\hline I & 1,08 & 0,048 & 4,4 & 0,034 & 3,1 \\
\hline II & 3,48 & 0,184 & 5,3 & 0,123 & 3,5 \\
\hline III & 10,1 & 0,506 & 5,0 & 0,353 & 3,5 \\
\hline IV & 19,7 & 0,921 & 4,7 & 0,652 & 3,3 \\
\hline V & 17,5 & 3,02 & 17,3 & - & - \\
\hline VI & 35,8 & 19,4 & 54,1 & 13,3 & 37,3 \\
\hline VII & 43,3 & 20,8 & 48,0 & 14,0 & 32,3 \\
\hline
\end{tabular}

a) Im Luftstrom von 90,5 mi/min; Desorpt.-dauer: $T$-I bis T - IV $99 \mathrm{~h}, \mathrm{~T}-\mathrm{V} 164 \mathrm{~h}, \mathrm{~T}-\mathrm{VI} 164 \mathrm{~h}, \mathrm{~T}-\mathrm{VII} 216 \mathrm{~h}$

b) Freiland/Nachdesorption: T - I bis T-IV $28 \mathrm{~d} / \mathrm{O} h, T-V I 63 \mathrm{~d} / 72 \mathrm{~h}, \mathrm{~T}-\mathrm{VII} 89 \mathrm{~d} / 72 \mathrm{~h}$

c) $\left.s_{\mathrm{e}}[\%] 4,4 \longleftrightarrow 23,4(n=5-18) ;{ }^{d}\right)$ Bezogen auf die TCE-Konzentration in den Nadeln zu Begasungsende

Tabelle 4: TCE-Konzentrationen in verschiedenen Bestandteilen der Fichtenzweige, die durch einmalige bzw. mehrfach wiederholte Eindosierung von gasförmiger Testsubstanz in die Versuchsvorrichtung begast und anschließend im definierten Luftstrom sowie an freier Luft desorbiert wurden (TCE-Äquivalentkonzentrationen)

\begin{tabular}{|c|c|c|c|c|c|}
\hline \multirow{3}{*}{$\begin{array}{l}\text { TCE-Begasungsversuch } \\
\text { (vgl. Tab. 1) } \\
\text { Nr. T- }\end{array}$} & \multicolumn{2}{|c|}{ Belasteter Zweigteil } & \multicolumn{3}{|c|}{ Unbelasteter Zweigteil } \\
\hline & Rinde/Bast & Holz & Nadeln & Rinde/Bast & Holz \\
\hline & $\left.[n g / g]^{a}\right)$ & $\left.[n g / g]^{a}\right)$ & $\left.[n g / g]^{b}\right)$ & $\left.[n g / g]^{b}\right)$ & $\left.[n g / g]^{b}\right)$ \\
\hline 1 & - & 1,6 & - & - & 0,5 \\
\hline II & - & 8,2 & - & $\rightarrow$ & 1,5 \\
\hline III & - & 25,9 & - & - & 3,8 \\
\hline IV & 608 & 13,4 & - & - & 2,6 \\
\hline V & - & - & - & - & - \\
\hline VI & 5042 & 113,3 & 16,1 & 188,0 & 3,3 \\
\hline VII & 3862 & 189,2 & 9,0 & 101,4 & 10,6 \\
\hline
\end{tabular}

a) $s_{\theta}[\%] 4,5 \leftrightarrow 44,2$ (n= 5 oder 6$) ; \quad$ b) $s_{\theta}[\%] 4,7 \leftrightarrow 86,0(n=4-6$ ) 


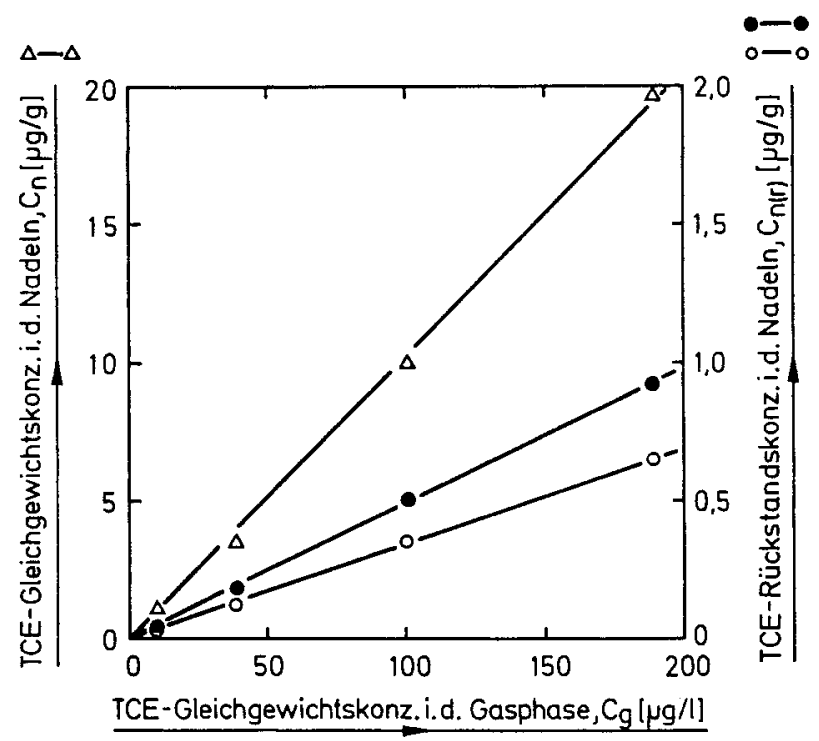

- - nach Desorption im konst. Luftstrom (90,5 ml/min; 99 h) 0 - 0 nach zusätzlicher Desorption unter Freilandbedingungen (28 d)

Abb. 1: Gleichgewichts- und Restkonzentrationen an Tetrachlorethen (TCE) in den Nadeln der begasten Fichtenzweige als Funktion der TCE-Gleichgewichtskonzentration in der Begasungsluft (Versuch Nr. T - I bis T - IV)

Gasphasenkonzentrationen gültigen Proportionalbereich der Ad-/Absorption befand; d.h., Gleichung (1) muß auch dann gültig sein, wenn die Atmosphäre im Belastungsgefäß - ähnlich wie die an sog. Reinluftstandorten - nur wenige ng TCE pro l Luft enthält.

Die zugehörigen Desorptionskurven in Abb. 2 geben kumulativ die mit der Zeit von den Nadeln und übrigen Zweigstellen an den konstanten Luftstrom abgegebenen TCE-Mengen wieder. Die Desorption des TCE erfolgt zunächst sehr schnell, nach längeren Zeiten dagegen sehr langsam. Nimmt man an, daß die Abnahme der Konzentration an TCE in den verschiedenen Teilen eines belasteten Zweiges proportional $\mathrm{zu}$ den dort vorliegenden TCEKonzentrationen ist, so läßt sich der beobachtete Desorptionsvorgang in zwei parallel verlaufende Teilprozesse unterschiedlicher Geschwindigkeit zerlegen. Diese Teilprozesse sind die Desorption des TCE

1. von den Nadeln,

2. von den übrigen Zweigteilen (Rinde, Bast, Holz). Dementsprechend wird die insgesamt $\mathrm{z} . \mathrm{Zt}$. $\mathrm{t}$ desorbierte TCE-Menge nach Gleichung (2) erhalten, wobei $N$ die Menge an TCE in den Nadeln und $Z$ die in den übrigen Teilen des Zweiges zur Zeit $t_{0}$ der Desorption ist:

$$
m_{d}=N\left(1-e^{-k_{1}{ }^{2}}\right)+Z\left(1-e^{-k_{2} t^{2}}\right)
$$

Gleichung (2): Gesamte, durch den versuchstechnisch vorgegebenen Luftstrom in der Zeit $t$ vom Fichtenzweig desorbierte TCE-Menge $m_{d}$

Die ausgezogenen Kurven in $\rightarrow$ Abb. 2 sind entsprechend der Gleichung (2) unter Verwendung der angegebenen analytischen Daten und der Geschwindigkeitskonstanten berechnet worden. Neben der erfolgreichen Kurvenanpassung ist vor allem die folgende Feststellung bemerkenswert:

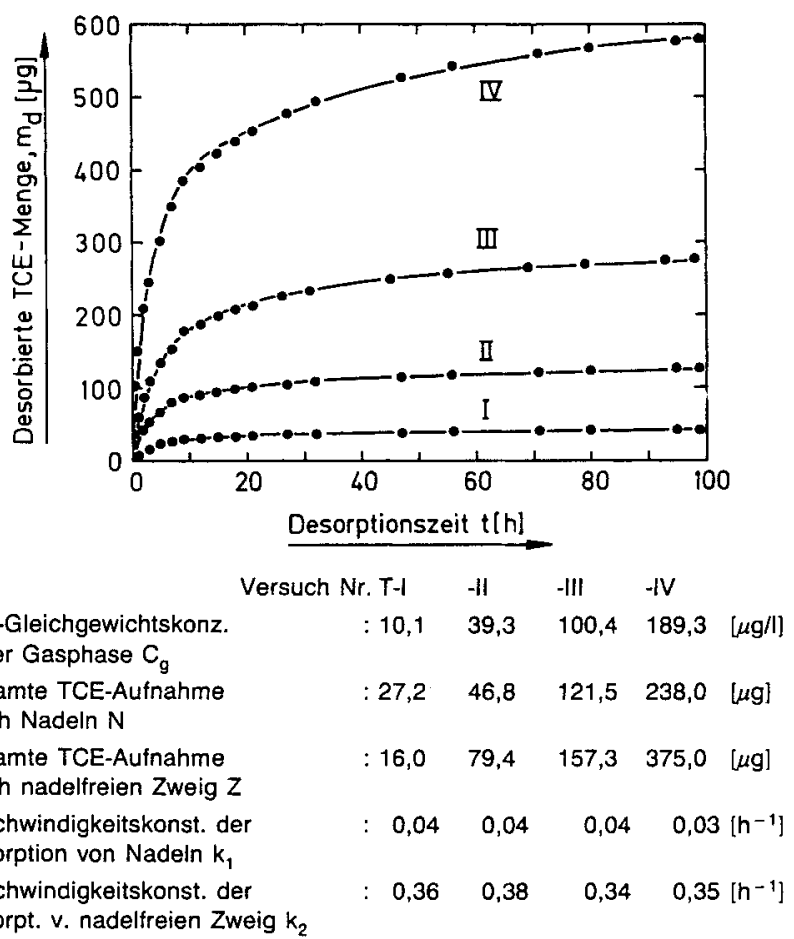

Abb. 2: Desorption des Tetrachlorethens (TCE) von verschieden stark begasten Fichtenzweigen im konstanten Luftstrom. Desorptionsbedingungen: $90,5 \mathrm{ml} \mathrm{Luft} / \mathrm{min}$ bei $22{ }^{\circ} \mathrm{C} \pm$ $1{ }^{\circ} \mathrm{C}$ (Versuch Nr. T - I bis T - IV)

Die Geschwindigkeitskonstanten für die Desorption des TCE von den Nadeln $\left(k_{1}\right)$ und den nadelfreien Teilen $\left(k_{2}\right)$ eines Fichtenzweiges unterscheiden sich um eine Größenordnung; d.h., bei gleicher ad-/absorbierter TCE-Menge würde die Desorption von den nadelfreien Zweigteilen etwa $10 \mathrm{mal}$ so schnell wie die von den Nadeln selbst erfolgen.

Die nach ca. 100stündiger Desorption im Luftstrom $(90,5 \mathrm{ml} / \mathrm{min})$ noch in den Nadeln der verschieden stark belasteten Zweige vorliegenden Rückstandskonzentrationen an TCE ergeben die mittlere Gerade in $\rightarrow$ Abb. 1. Wie die untere Kurve in Abb. 1 zeigt, nahmen die TCEKonzentrationen in den Nadeln der verschieden stark belasteten Zweige während des anschließenden, 28tägigen Aufenthalts der Versuchsfichten in freier Atmosphäre weiter deutlich ab. Aus dem Anstieg dieser Geraden erhält man eine Geschwindigkeitskonstante für die Desorption der TCERestmengen aus Nadeln in freier Atmosphäre von $5,2 \cdot 10^{-4} \mathrm{~h}^{-1}$. Sie ist im Vergleich zu der, die für die Desorption der Hauptmenge an TCE aus denselben Nadeln im konstanten Luftstrom berechnet wurde, um ca. 2 GröBenordnungen kleiner.

Nach Abschluß der Desorption (d.h. nach 99 h im Luftstrom von $90,5 \mathrm{ml} / \mathrm{min}+28 \mathrm{~d}$ in freier Atmosphäre) lagen $\mathrm{im}$ Mittel noch 3,4\% $\left(\mathrm{s}_{\mathrm{e}}= \pm 0,18, \mathrm{n}=4\right) \mathrm{der}$ TCE-Mengen, die während der unterschiedlich starken 24stündigen Begasungen aufgenommen wurden, als schwer oder nicht mehr desorbierbare Rückstände in den Nadeln der Fichtenzweige vor (vgl. $\rightarrow$ Tabelle 3). Wie auch die Gegenüberstellung der TCE-Konzentrationen in den unterschiedlich begasten Nadeln vor und nach der Desorption 
zeigt ( $\rightarrow$ Tabelle 3), bestand, wie bereits erwähnt, unter den gewählten Desorptionsbedingungen zwischen der TCE-Ausgangs- und TCE-Rückstandskonzentration in den Nadeln eine direkte Proportionalität; d.h. die prozentualen Anteile an TCE, die nach Abschluß der Desorptionsversuche noch in den unterschiedlich stark begasten Nadeln vorlagen, waren gleich, unabhängig von der Höhe der TCE-Anfangskonzentrationen $c_{n}$.

Im Begasungsversuch T-VI bzw. T-VII $(\rightarrow$ Tabelle 1$)$ wurden jeweils zu den Zeitpunkten 0,24 , und 48 h gleich große Portionen an ${ }^{14} \mathrm{C}$-TCE in die mit einem Fichtenzweig versehene Glasküvette eindosiert ( $3 \mathrm{fach}$ wiederholte TCE-Applikation). Zur Charakterisierung des Begasungsverlaufs wurden der Glasküvette zu bestimmten Zeiten jeweils mehrere Luftproben entnommen und diese radioanalytisch auf den Gehalt an TCE untersucht.

Wie aus Abb. 3 hervorgeht, stieg bei jeder Eindosierung die TCE-Konzentration in der Innenatmosphäre der Glasküvette spontan an (nicht ausgefüllte Zeichen), um danach schnell auf die für das Kontaktsystem „Fichtenzweig/Luft“ typische TCE-Gleichgewichtskonzentration abzusinken; d.h., durch die wiederholte Stoffapplikation wurde die TCE-Gleichgewichtskonzentration im Luftraum der Begasungsküvette stufenweise jeweils um den theoretisch erwarteten Betrag erhöht.

Die Kinetik der Desorption des TCE, das vorher bei der stufenweisen Anhebung der TCE-Gleichgewichtskonzentration im Begasungsraum innerhalb $72 \mathrm{~h}$ vom Fichtenzweig aufgenommen wurde, entsprach gleichfalls dem bereits oben mitgeteilten mathematischen Formalismus. Demnach wird auch die in Abb. 4 dargestellte, aus dem Versuch Nr. T-VI stammende TCE-Desorptionskurve durch Gleichung (2) gut beschrieben. Es entsprach somit auch den Erwartungen, daß die TCE-Menge, die der in dieser Weise begaste Fichtenzweig während der Desorption wieder an die Luft abgab, ca. $3 \mathrm{mal}$ so groß war wie die Menge, die der Zweig aus Versuch Nr. T - V freisetzte, der

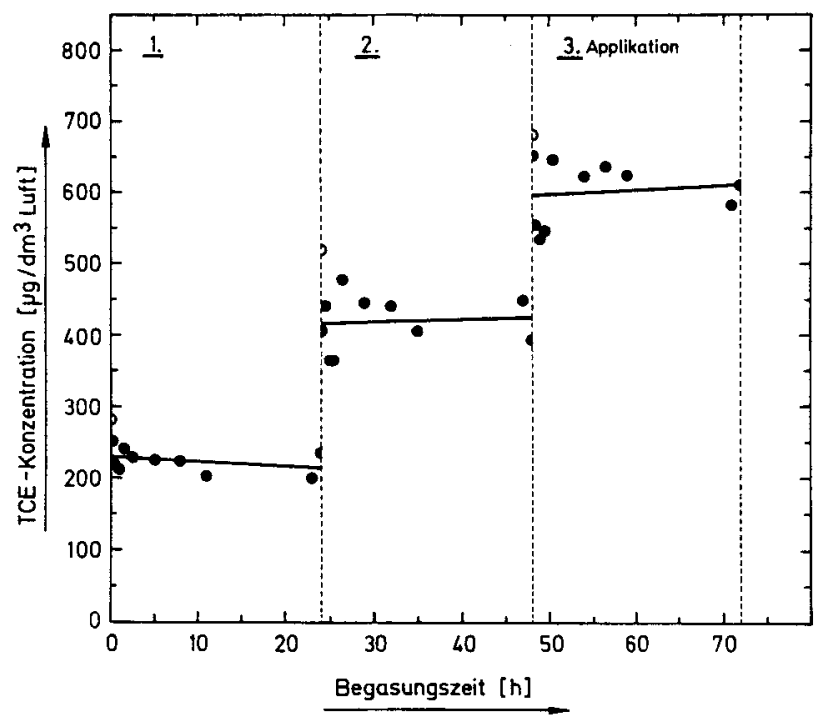

Abb. 3: Stufenweiser Anstieg der TCE-Gleichgewichtskonzentration in der Luft der mit einem Fichtenzweig beschickten Begasungsvorrichtung bei mehrfach wiederholter Eindosierung gleicher Portionen an ${ }^{14} \mathrm{C}-\mathrm{TCE}$ (Versuch Nr. T - VI)

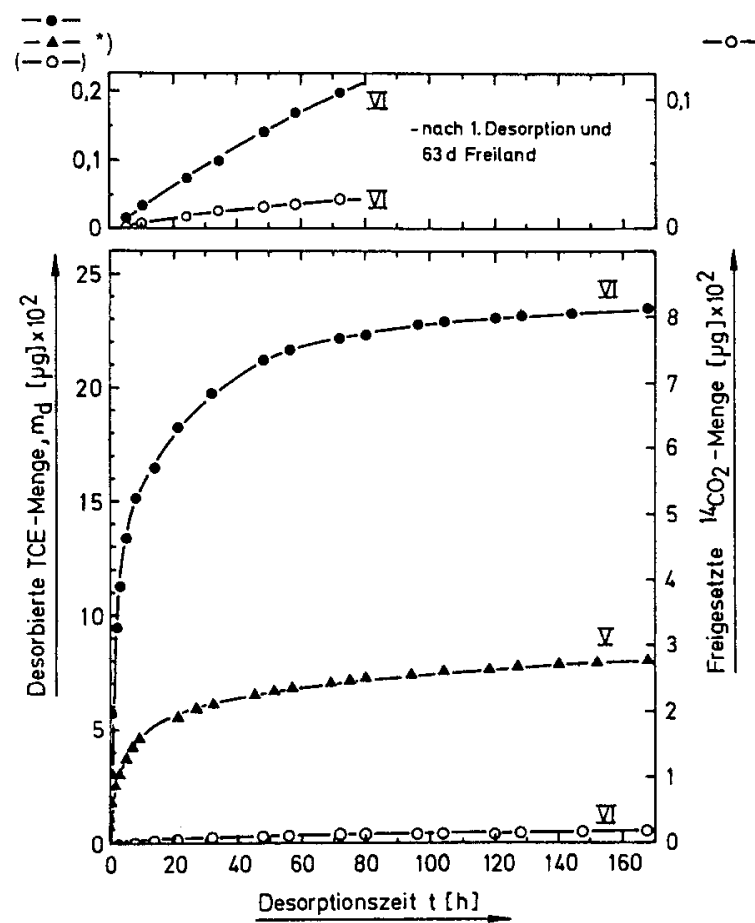

$\left.{ }^{*}\right)$ zu ${ }^{14} \mathrm{CO}_{2}$ abgebaute Menge an ${ }^{14} \mathrm{C}$-markiertem TCE

Abb. 4: Desorption des Tetrachlorethens (TCE) sowie Folgeprodukte von unterschiedlich begasten Fichtenzweigen im konstanten Luftstrom $\left(90,5 \mathrm{ml} \mathrm{Luft} / \mathrm{min}\right.$ bei $\left.22^{\circ} \mathrm{C} \pm 1{ }^{\circ} \mathrm{C}\right)$

- Versuch Nr. T-V: nach einer Begasungszeir von $48 \mathrm{~h}$ bei einer mitcleren TCE-Gleichgewichtskonzentration von $220,0 \mu \mathrm{g} / \mathrm{dm}^{3}$ Luft

- Versuch Nr. T-VI: nach einer Begasungszeit von $72 \mathrm{~h}$ bei einer stufenweise erreichten, mittleren TCEGleichgewichtskonzentration von $602,5 \mu \mathrm{g} /$ $\mathrm{dm}^{3}$ Luft; nach $63 \mathrm{~d}$ Freiland wurde die Desorption des TCE vom begasten Zweig fortgesetzt

nach der Applikation nur einer TCE-Portion 48 h lang ausgesetzt war.

Darüber hinaus zeigen die $\rightarrow$ Tabellen $3+4$, daß mit wachsender Begasungsdauer der prozentuale Anteil an nur schwer oder nicht mehr desorbierbaren TCE-Rückständen in den begasten Nadeln deutlich zunahm.

Außerdem stellten wir fest, daß die vorher TCE-begasten Fichtenzweige während des Desorptionsvorganges neben intaktem ${ }^{14} \mathrm{C}$-TCE auch meßbare Mengen an ${ }^{14} \mathrm{CO}_{2}$ an den Luftstrom abgaben. $\mathrm{Die}^{14} \mathrm{CO}_{2}$ Menge, die unter den gewählten Desorptionsbedingungen vom TCE-begasten Zweig aus Versuch Nr. T-VI freigesetzt wurde, ist als Funktion der Desorptionszeit $t$ im unteren Teil der Abb. 4 dargestellt. Wie die Kurven im oberen Teil belegen, setzte der Zweig auch nach 164stündiger Desorption und einem anschließenden 63tägigen Aufenthalt an freier Luft weitere meßbare Mengen an ${ }^{14} \mathrm{C}$-TCE und ${ }^{14} \mathrm{CO}_{2}$ frei. Wir haben deutliche Hinweise, daß zwei Pilzarten, die ausschließlich auf/in der Rinde ( + Bast) und nicht auf den Nadeln der gesunden Versuchsfichten siedeln, das dort ad-/absorbierte ${ }^{14} \mathrm{C}$-TCE zu einem geringen Anteil in ${ }^{14} \mathrm{CO}_{2}$ überführten.

Die Begasung vitaler Fichtenzweige mit [Carbonyl- ${ }^{14} \mathrm{C}$ ] 
Furfural $\left({ }^{14} \mathrm{C}-\mathrm{F}\right)$ wurde nach demselben Prinzip wie die mit ${ }^{14} \mathrm{C}$-TCE durchgeführt. Jedoch verlief die Ad-/Absorption des Furfurals durch Fichtenzweige völlig anders als die des Tetrachlorethens.

Dieses unterschiedliche Verhalten wird deutlich sichtbar beim Vergleich von Abb. 5 mit der entsprechenden $\rightarrow$ Abb. 3 für TCE. Im Gegensatz zum TCE, für das sich nach jeder erneuten Applikation in der Innenatmosphäre der Begasungsküvette eine höhere Gleichgewichtskonzentration einstellte, wurde beim Furfural auch noch nach mehrfach wiederholter Applikation jeder Neuzugang vom Zweig vollständig aufgenommen. Die geringe Erhöhung der Radioaktivitätskonzentration, die nach der 2. und auch 3. Eindosierung von ${ }^{14} \mathrm{C}-\mathrm{F}$ im Begasungsraum beobachtet wurde, ist auf die Bildung von radioaktivem Kohlendioxid zurückzuführen.

Besonders deutlich zutage tritt der Unterschied, der zwischen dem Übertritt von Furfural und dem von TCE aus der Gasphase auf Fichtenzweige bestand, beim Vergleich von $\rightarrow$ Tabelle $5+6$ mit Tabelle $3+4$. Die Gegenüberstellung zeigt, daß die ${ }^{14} \mathrm{C}-\mathrm{F}-\mathrm{Konzentrationen}$ in allen Bestandteilen der behandelten Fichtenzweige stets um mehr als eine Zehnerpotenz über den vergleichbaren ${ }^{14} \mathrm{C}$-TCEKonzentrationen lagen. Ein durch Zahlen belegter quantitativer Vergleich zwischen der Aufnahme von Furfural und der von TCE durch Fichtenzweige ist jedoch nicht möglich, $\mathrm{da}$ das in die Begasungsküvette eindosierte gasförmige ${ }^{14} \mathrm{C}$ $\mathrm{F}$, das wegen der verwendeten Applikationstechnik ebenso

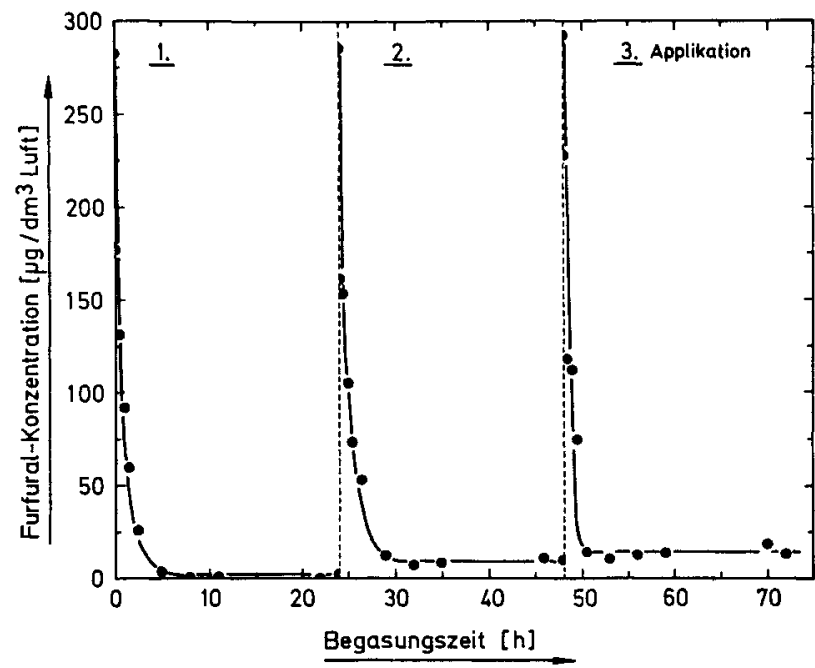

Abb. 5: Abfall der Furfural-Konzentration in der Luft der mit einem Fichtenzweig beschickten Begasungsvorrichtung nach jeder erneuten Eindosierung einer gleich großen Menge an ${ }^{14} \mathrm{C}$ Furfural (Versuch Nr. F-IV)

Tabelle 5: Furfural-Konzentrationen in den Nadeln der Fichtenzweige, die durch einmalige bzw. mehrfach wiederholte Eindosierung von gasförmiger Testsubstanz in die Versuchsvorrichtung begast und anschließend im definierten Luftstrom sowie an freier Luft desorbiert wurden (Furfural-Äquivalentkonzentrationen)

Furfural-Begasungsversuch (vgl. Tab. 2)
Konzentration an Furfural in Fichtennadeln

nach 1. Desorptionb) (löslicher Teil)a) nach 1 . Desorption und

Freiland (Fr.) bzw. 1. Desorp., Fr. U. Nachdesorption ${ }^{c}$ )
Nr. F-

\begin{tabular}{cccc} 
Nr. F $-~$ & $\left.[\mu \mathrm{g} / \mathrm{g}]^{\mathrm{d}}\right)$ & $\left.[\mu \mathrm{g} / \mathrm{g}]^{\mathrm{d}}\right)$ & $\left.[\mu \mathrm{g} / \mathrm{g}]^{\mathrm{d}}\right)$ \\
\hline I & 400 & 719 & 971 \\
II & 209 & 544 & 443 \\
III & 287 & 904 & 595 \\
IV & 714 & 1832 & 1969 \\
V & 329 & 852 & 832 \\
VI & 285 & - & - \\
\hline
\end{tabular}

$\left.[\mu \mathrm{g} / \mathrm{g}]^{\mathrm{d}}\right)$

$\left.[\mu \mathrm{g} / \mathrm{g}]^{\mathrm{d}}\right)$

\begin{tabular}{cccc} 
Nr. F $-~$ & $\left.[\mu \mathrm{g} / \mathrm{g}]^{\mathrm{d}}\right)$ & $\left.[\mu \mathrm{g} / \mathrm{g}]^{\mathrm{d}}\right)$ & $\left.[\mu \mathrm{g} / \mathrm{g}]^{\mathrm{d}}\right)$ \\
\hline I & 400 & 719 & 971 \\
II & 209 & 544 & 443 \\
III & 287 & 904 & 595 \\
IV & 714 & 1832 & 1969 \\
V & 329 & 852 & 832 \\
VI & 285 & - & - \\
\hline
\end{tabular}

a) Berechnet anhand der Radioaktivitätsmengen, die bei längerem Kontakt (bis zu $114 \mathrm{~d}$ ) aus den belasteten Nadeln in Tetralin übertrateı

b) Im Luftstrom von $90,5 \mathrm{ml} / \mathrm{min}$; Desorpt.-dauer F-I $122 \mathrm{~h}, \mathrm{~F}-1 \mathrm{II} 117 \mathrm{~h}, \mathrm{~F}-\mathrm{III} 121 \mathrm{~h}, \mathrm{~F}-\mathrm{IV} 168 \mathrm{~h}, \mathrm{~F}-\mathrm{V} 128 \mathrm{~h}$

c) Freiland/Nachdesorption: $F-1, F-\|$ und $F-I I I 56$ d/O h, F - IV $63 \mathrm{~d} / 72 \mathrm{~h}, \mathrm{~F}-\mathrm{V} 63 \mathrm{~d} / 48 \mathrm{~h}$

d) $s_{e}[\%] 9,4 \longleftrightarrow 34,9(n=5-20)$

Tabelle 6: Furfural-Konzentrationen in verschiedenen Bestandteilen der Fichtenzweige, die durch einmalige bzw. mehrfach wiederholte Eindosierung von gasförmiger Testsubstanz in die Versuchsvorrichtung begast und anschließend im definierten Luftstrom sowie an freier Luft desorbiert wurden (Furfural-Äquivalentkonzentrationen)

\begin{tabular}{|c|c|c|c|c|c|}
\hline \multirow{3}{*}{$\begin{array}{c}\text { Furfural-Begasungs- } \\
\text { versuch (vgl. Tab. 2) } \\
\text { Nr. F- }\end{array}$} & \multicolumn{2}{|c|}{ Belasteter Zweigteil } & \multicolumn{3}{|c|}{ Unbelasteter Zweigteil } \\
\hline & Rinde/Bast & Holz & Nadeln & Rinde/Bast & Holz \\
\hline & $\left.[\mu \mathrm{g} / \mathrm{g}]^{a}\right)$ & $\left.[\mu \mathrm{g} / \mathrm{g}]^{\mathrm{a}}\right)$ & $\left.[\mu \mathrm{g} / g]^{b}\right)$ & $\left.[\mu \mathrm{g} / \mathrm{g}]^{\mathrm{b}}\right)$ & $\left.[\mu \mathrm{g} / \mathrm{g}]^{b}\right)$ \\
\hline | & 64,7 & 1,17 & - & 7,36 & 0,53 \\
\hline 11 & 25,7 & 0,71 & - & 1,36 & 0,22 \\
\hline III & 155,4 & 5,10 & - & 4,43 & 0,97 \\
\hline IV & 157,9 & 18,3 & - & 0,38 & 0,051 \\
\hline V & 36,9 & 0,49 & 0,042 & 0,28 & 0,024 \\
\hline $\mathrm{V} 1$ & - & - & - & - & - \\
\hline
\end{tabular}

a) $s_{e}[\%] 11,2 \longleftrightarrow 65,6(n=5-10) ; \quad$ b) $s_{\theta}[\%] \quad 4,6 \longrightarrow 33,4(n=5-10)$ 
wie das ${ }^{14} \mathrm{C}$-TCE zunächst auf das Ende des eingeschlossenen Zweiges traf, bereits dort - und das im Gegensatz zum ${ }^{14} \mathrm{C}$-TCE - weitgehend ad-/absorbiert wurde und folglich nur in deutlich geringerer Konzentration die stammnahen Zweigteile erreichte; d.h., im Falle des Teststoffes ${ }^{14} \mathrm{C}-\mathrm{F}$ wurden die Fichtenzweige regional unterschiedlich begast. Dies war uns zur Zeit der Probenahmen, die willkürlich in allen Bereichen eines Zweiges vorgenommen wurden, noch nicht bekannt. Es ist zu vermuten, daß auch die Diskrepanzen zwischen den in $\rightarrow$ Tabelle 5 angeführten ${ }^{14} \mathrm{C}-\mathrm{F}$ Konzentrationen in Nadeln hier ihren Ursprung haben (beachte auch Fußnote a) unter Tabelle 5).

Der in Abb. 6 als Beispiel dargestellte Desorptionsablauf weist vor allem darauf hin, daß die mit ${ }^{14} \mathrm{C}$-F begasten Fichtenzweige während des Desorptionsvorganges in dominierendem Maße radioaktives Kohlendioxid $\left({ }^{14} \mathrm{CO}_{2}\right)$ freisetzten. Diese bereits bei der Desorption von TCE-begasten Fichtenzweigen am Rande beobachtete und nun bei der Desorption der mit Furfural behandelten Zweige in den Mittelpunkt tretende Erscheinung veranlaßte uns, nach den Mikroorganismen zu suchen, die unter den Desorptionsbedingungen das [Carbonyl $-{ }^{14} \mathrm{C}$ ] Furfural sowie das $\left[1,2-{ }^{14} \mathrm{C}\right.$ ] Tetrachlorethen in ${ }^{14} \mathrm{CO}_{2}$ überführten. Dabei stellten wir fest, daß ausschließlich die Rinde ( + Bast) und nicht die gesunden Nadeln der Versuchsfichten durch nur zwei unterschiedliche, in Gemeinschaft lebende Pilze besiedelt waren. (Sie wurden inzwischen von KRISTEN und PETERSEN, Botanisches Institut der Universität Hamburg, isoliert und sollen in Zusammenarbeit mit uns auf ihre Abbauleistung für Furfural und TCE untersucht werden.)

Nach Abschluß der mikrobiologischen Überprüfung der Versuchsfichten wurden parallel zueinander zwei weitere

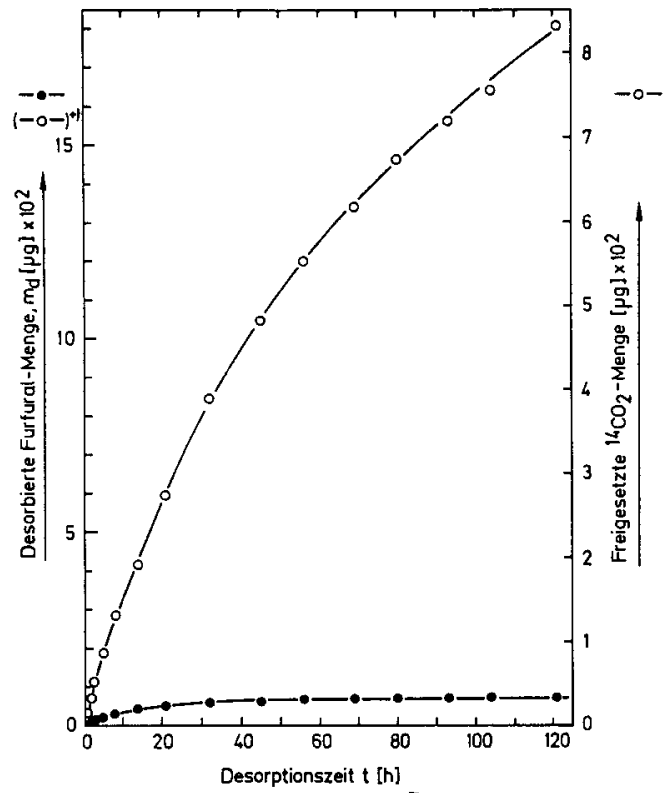

$+)$

zu ${ }^{14} \mathrm{CO}_{2}$ abgebaute Menge an ${ }^{14} \mathrm{C}$-markiertem Furfural

Abb. 6: Desorption des Furfurals sowie Folgeprodukte von einem begasten Fichtenzweig im konstanten Luftstrom $(90,5 \mathrm{ml}$ Luft/min bei $22^{\circ} \mathrm{C} \pm 1{ }^{\circ} \mathrm{C}$ )

- Versuch Nr. F-III; nach einer Begasungszeit von 72 h, wobei zu den Zeitpunkten 0,24 und $48 \mathrm{~h}$ jeweils $7,19 \mathrm{mg}$ dampfförmiges ${ }^{14} \mathrm{C}$-Furfural in die Begasungsvorrichtung appliziert wurden
Begasungen von Zweigen mit gleich großen Mengen an ${ }^{14} \mathrm{C}-\mathrm{F}$ vorgenommen (Versuch $\mathrm{Nr} \mathrm{F}-\mathrm{V}$ und $\mathrm{F}-\mathrm{VI}$; vgl. $\rightarrow$ Tabelle 2). Unmittelbar nach Begasungsende wurde der Zweig aus Begasungsversuch Nr. F-VI zunächst von allen Nadeln befreit; während der dazu benötigten Zeit blieb der begaste $Z$ weig aus Versuch F-V unberührt an freier Luft. Erst danach wurden die Zweige gleichzeitig in die Glasrohre von zwei autark arbeitenden Desorptionseinheiten geschoben und dort - wie oben beschrieben - unter vergleichbaren Bedingungen im definierten Reinluftstrom behandelt. Die Messung der desorbierten, in den Absorbersystemen der Desorptionseinheit aufgetrennten radioaktiven Stoffe zeigte, daß die Freisetzung von ${ }^{14} \mathrm{CO}_{2}$ durch den nadelfreien und die durch den unberührt gelassenen Fichtenzweig hinsichtlich Verlauf und Menge gut übereinstimmten. So wurden z.B. vom nadelfreien Zweig $73 \mu \mathrm{g}$ und vom unversehrten $62 \mu \mathrm{g}{ }^{14} \mathrm{CO}_{2}$ in $70 \mathrm{~h}$ an den Luftstrom abgegeben.

Diese Befunde bestärken uns in der Ansicht, daß die oben erwähnten Pilze das adsorptiv auf/in Rinde und Bast angereicherte Furfural nutzten und zumindest z.T. in Kohlendioxid überführten.

\section{Resümee und Schlußbetrachtung}

Die Untersuchungsergebnisse gestatten im Hinblick auf das Verhalten von Tetrachlorethen (TCE) im Kontaktsystem "Luft/Fichtenzweig bzw. Fichtennadeln“ folgende Aussagen:

- Das Verteilungsgleichgewicht für TCE im Kontaktsystem "Luft/Fichtenzweig" stellte sich unter Versuchsbedingungen innerhalb 1 Stunde ein.

- Eine signifkante Verschiebung der Lage des TCEVerteilungsgleichgewichts in Richtung Fichtenzweig wurde unter den Versuchsbedingungen nicht beobachtet; d.h., die TCEKonzentration in der Luft blieb nach der Gleichgewichtscinstellung über den Rest der Versuchszeit praktisch konstant.

- Der Zusammenhang zwischen der TCE-Konzentration in der Luft und der in Fichtennadeln wird durch eine aus den experimentellen Daten abgeleitete mathematische Funktion gut beschrieben, ist also berechenbar.

- Die Geschwindigkeitskonstanten für die Desorption des TCE von den Nadeln und den nadelfreien Teilen eines Fichtenzweiges im Luftstrom unterscheiden sich um ca. eine Größenordnung; d.h., bei gleicher ad-/absorbierter TCE-Menge wird die Desorption von den nadelfreien Zweigteilen etwa $10 \mathrm{mal}$ so schnell wie die von den Nadeln selbst erfolgen.

- Die insgesamt durch einen Luftstrom von einem TCE-belasteten Fichtenzweig desorbierte TCE-Menge ist ebenfalls durch eine mathematische Funktion berechenbar.

- Nach 24stündiger TCE-Begasung und Abschluß einer anschließenden, 99stündigen Desorption lagen - unabhängig von der Höhe der TCE-Anfangskonzentration in den unterschiedlich stark belasteten Fichtennadeln - noch ca. 3,4\% der ursprünglich vorhandenen TCE-Menge als schwerer bzw. nicht mehr desorbierbarer Anteil in den Nadeln vor. Dieser in den Nadeln festgelegte TCEAnteil wächst deutlich mit Verlängerung der Begasungszeit.

- Die Geschwindigkeitskonstante für die Desorption der Restmengen an mobilen TCE aus Nadeln in freier Atmosphäre betrug 5,2 . $10^{-4} \mathrm{~h}^{-1}$. Sie ist im Vergleich zu der, die für die Desorption der Hauptmenge an TCE aus denselben Nadeln im konstanten Luftstrom berechnet wurde, um ca. 2 Größenordnungen kleiner.

- Fichtenzweige sind für luftgetragenes TCE (im Gegensatz zu anderen organischen Stoffen) keine unmittelbare Senke. 
Tabelle 7: Gegenüberstellung der zur Begasung eingesetzten und der nach Desorption in Labor und Freilanda) noch in den Nadeln vorliegenden TCE- bzw. Furfural-Mengen ${ }^{b}$ )

\begin{tabular}{|c|c|c|c|c|c|c|c|c|}
\hline \multirow{3}{*}{$\begin{array}{l}\text { Versuch } \\
\text { Nr. T-/F- }\end{array}$} & \multicolumn{4}{|c|}{ Tetrachlorethen (TCE) } & \multicolumn{4}{|c|}{ Furfural } \\
\hline & \multirow{2}{*}{$\begin{array}{l}\text { Applizierte } \\
\text { Menge } \\
\text { [mg] }\end{array}$} & \multirow{2}{*}{$\begin{array}{c}\text { Begasungs- } \\
\text { zeit } \\
{[h]}\end{array}$} & \multicolumn{2}{|c|}{$\begin{array}{l}\left.\text { Rückstand }{ }^{b}\right) \text { i.d. } \\
\text { Nadelmasse }\end{array}$} & \multirow{2}{*}{$\begin{array}{l}\text { Applizierte } \\
\text { Menge } \\
\text { [mg] }\end{array}$} & \multirow{2}{*}{$\begin{array}{c}\text { Begasungs- } \\
\text { zeit } \\
{[\mathrm{h}]}\end{array}$} & \multicolumn{2}{|c|}{$\begin{array}{l}\text { Rückstand b) i.d. } \\
\text { Nadelmasse }\end{array}$} \\
\hline & & & {$[\mu \mathrm{g}]$} & {$[\%]$} & & & [mg] & {$[\%]$} \\
\hline 1 & 0,357 & 24 & 0,53 & 0,15 & 7,19 & 24 & 6,60 & 92 \\
\hline ॥ & 1,43 & 24 & 1,75 & 0,12 & 7,19 & 24 & 5,27 & 73 \\
\hline III & 3,57 & 24 & 4,48 & 0,13 & 21,57 & 72 & 11,7 & 54 \\
\hline IV & 7,15 & 24 & 6,39 & 0,09 & 21,57 & 72 & 20,1 & 93 \\
\hline V & 7,15 & 48 & 25,1 & 0,35 & 10,78 & 24 & 9,65 & 90 \\
\hline $\mathrm{VI}$ & $\left.21,45^{c}\right)$ & 72 & 258,3 & 1,29 & - & - & - & - \\
\hline VII & $\left.21,45^{c}\right)$ & 72 & 296,4 & 1,38 & - & - & - & - \\
\hline
\end{tabular}

a) Vgl. Fußnote a) und b) unter Tab. 3 bzw. Fußnote b) und c) unter Tab. 5; b) Die zur gefundenen Radioaktivität äquivalente Stoffmenge; appl. Menge = 100 \% 9) In 3 gleichen Portionen zu den Zeitpunkten $t_{1}=0 \mathrm{~h}, t_{2}=24 \mathrm{~h}$ und $t_{3}=48 \mathrm{~h}$ in die Begasungsvorrichtung appliziert

Inwieweit diese auf das Verhalten von Tetrachlorethen im Kontaktsystem "Luft/Fichtenzweig bzw. -nadeln“ zutreffenden Aussagen auch auf die übrigen halogenierten aliphatischen Kohlenwasserstoffe übertragbar sind, muß durch weitere Experimente überprüft werden (FRANK et al., 1985; GRIMMER et al., 1986; BowEN et al., 1953; SCHLICHT et al., 1972; FrANK et al., 1986; DEBUS, SCHRÖDER 1989).

Die Versuche mit Furfural zeigen:

Das gasförmige mit der Luft zugeführte Furfural tritt schnell und quantitativ auf den Fichtenzweig - insbesondere auf dessen Nadeln - über und ist danach nur noch zu einem außerordentlich geringen Anteil wieder desorbierbar. So sind Fichtenzweige bzw. -nadeln für luftgetragenes Furfural eine totale Senke. Dieser Unterschied im Verhalten luftgetragener organischer Stoffe gegenüber Pflanzen tritt besonders deutlich zutage beim Vergleich der FurfuralAnteile mit den entsprechenden TCE-Anteilen in Tabelle 7.

Auch hier ist nicht bekannt, ob sich die anderen luftgetragenen natürlichen oder anthropogenen Aldehyde in ihrer Wechselwirkung mit Pflanzen ähnlich wie das von uns untersuchte Furfural verhalten. Völlig offen ist die Frage, über welche Mechanismen bereits ad-/ absorbierte atmogene Aldehyde in die physiologisch-biochemischen Prozesse der Pflanzen eintreten und dort wirken.

\section{Literatur}

Bowen, E. J.; RohatgI, K. K.: Photochemistry of anthracene. II. The photochemical reaction of anthracene with carbon tetrachloride. Discussions Faraday Soc. 14, 146-150 (1953)

Debus, R.; SCHRÖDER, P.: Wirkungen von Halon 1211 (Bromchlordifluormethan) auf Kresse, in: Halogenierte organische Verbindungen in der Umwelt - Herkunft, Messung, Wirkung, Abhilfemaßnahmen, VDI-Berichte 745, Band II, S. 563-572 (1989)

DOMMRÖSE, A. M.; FIGGE, K.: Qualitative und quantitative Bestimmung potentieller organischer Schadstoffe in der Luft des Standorts „Postturm" Forstamt Farchau/Ratzeburg) in: Das Forschungsprogramm Waldschäden am Standort "Postturm“, Forstamt Farchau/Ratzeburg, J. Bauch u. W. Michaelis, GKSS 88/E/55. - S. 61-7, Geesthacht 1988

DOMMRÖSE, A. M.; FIGGE, K.: Analysis of organic trace compounds in the atmosphere and the correlation between meteorologigal si- tuation and concentration of reference substances, Fresenius $\mathrm{Z}$. Anal. Chem. 332, 606-611 (1988)

Frank, H.; Frank, W.: Photochemical activation of chloroethenes leading to destruction of photosynthetic pigments. Experientia 42, $1267-1269$ (1986)

Frank, H.; Frank, W.: Chlorophyll-bleaching by atmospheric pollutants and sunlight - A probable cause of tree damages observed in recent years. Naturwissenschaften 72, 139-141 (1985)

Frank, H.; FrANK, W.: Photoaktivierung luftgetragener Chlorkohlenwasserstoffe. Nachr. Chem. Tech. Lab. 34, 15-20 (1985)

Grimmer, G.; SCHMidT, W.: Modellversuche zur Phytotoxizität von Halogenkohlenwasserstoffen, Angew. Chem. 98, 807-808 (1986)

HARNISCH, M.; MOCKEL, H. J.; SCHulze, G.: Relationship between $\log P_{O W}$ shake-flask values and capacity factors derived from reversed-phase high-performence liquid chromatography for $n$ alkylbenzenes and some OECD reference substances. J. Chromat. 282, 315 - 332 (1983)

KERLER, F.: Quantitative Bestimmung und Analyse von Sorptionsund Transportparametern der pflanzlichen Kutikula für lipophileorganische Verbindungen. Diss. Techn. Univ. München, Mai 1986.

KeRLER, F.; RIEDERER, M.; SCHÖNHERR, J.: Non-electrolyte permeability of plant cuticles: A critical evaluation of experimental methods. Physiol. Plant. 62, 599-602 (1984)

LIESENFELD, K.; FIGGE, K. DOMMRÖE, A. M.: Vorkommen organischer Stoffe in der Atmosphäre - Zusammenstellung und Bewertung von Analysenergebnissen, Schriftenreihe des Vereins für Wasser-, Boden- und Lufthygiene, Gustav Fischer Verlag, Stuttgart/New York (in Vorbereitung) (1990)

Miller, M. M.; Wasik, S. P.; Huang, G. L.; SHIU, W. Y.; Mackay, D.: Relationships between octanol-water partition coefficient and aqueous solubility. Environ. Sci. Technol. 19, $552-559$ (1985)

PEARSON C. R.: $C_{1}$ and $C_{2}$ halocarbons [in environmental chemistry], in: HutzinGER D. (ED.): Handbook of Environmental Chemistry, Vol. 3 B, p. 69-88, Springer Verlag, Berlin 1982

RIEDERER, M.; SCHÖNHERR, J.: Accumulation and transport of (2,4-dichlorophenoxy) acetic acid in plant cuticles: I. Sorption in the cuticular membrane and its compounds. Ecotoxicology and Environmental Safety 8, 236-247 (1984); II. Permeability of the cuticular membrane. Ecotoxicology and Environmental Safety 9 , $196-208$ (1985)

RiEDERER, M.; SCHÖNHERR, J.: Covalent binding of chlorophenoxyacetic acids to plant cuticles. Arch. Environ. Contam. Toxicol. 15, 97-105 (1986)

SCHLicht, G.; SCHULTE-Frohlinde, D.: Photodissociation of carbon tetrachloride sensitized by naphthalene derivatives. I. Radicalchain reaction in the system naphthalene-methanol-carbon tetrachloride. Photochem. Photobiol. 16, 183-188 (1972)

SHAFER, W. E.; SCHÖNHERR, J.: Accumulation and transport of phenol, 2-nitrophenol, and 4-nitrophenol in plant cuticles. Ecotoxicological and Environmental Safety 10, 239-252 (1985) 